\title{
TITLE:
}

\section{Quadrupolar order in isotropic Heisenberg models with biquadratic interaction}

\author{
$\operatorname{AUTHOR}(S)$ :
}

Harada, Kenji; Kawashima, Naoki

\section{CITATION:}

Harada, Kenji ...[et al]. Quadrupolar order in isotropic Heisenberg models with biquadratic interaction. Physical Review B 2002, 65(5): 052403.

\section{ISSUE DATE:}

2002-01-03

URL:

http://hdl.handle.net/2433/50294

RIGHT:

(C)2002 The American Physical Society 
PHYSICAL REVIEW B, VOLUME 65, 052403

\title{
Quadrupolar order in isotropic Heisenberg models with biquadratic interaction
}

\author{
Kenji Harada \\ Department of Applied Analysis and Complex Dynamical Systems, Kyoto University, Kyoto 606-8501, Japan \\ Naoki Kawashima \\ Department of Physics, Tokyo Metropolitan University, Tokyo 192-0397, Japan
}

(Received 25 September 2001; published 3 January 2002)

\begin{abstract}
Through quantum Monte Carlo simulation, we study the biquadratic interaction model with the SU(2) symmetry in two and three dimensions. The zero-temperature phase diagrams for the two cases are identical and exhibit an intermediate phase characterized by finite quadrupole moment, in agreement with mean-fieldtype arguments and semiclassical theory. In three dimensions, we demonstrate that the model in the quadrupolar regime has a phase transition at a finite temperature. In contrast to predictions by mean-field theories, the phase transition to the quadrupolar phase turns out to be of second order. We also examine the critical behavior in the two marginal cases with the SU(3) symmetry.
\end{abstract}

DOI: 10.1103/PhysRevB.65.052403

PACS number(s): 75.40.Mg, 75.10.Jm, 75.10.Dg, 75.30.Et

Spin interactions of order higher than second have been discussed for many years. ${ }^{1,2}$ There are various sources of the high-order terms. For example, they may arise from the effect of crystalline fields or the high-order perturbations of electron exchanges. These high-order terms were identified or speculated to be responsible for some of the phase transitions observed in various magnetic materials. ${ }^{3-9}$ In contrast to second-order or bilinear interaction models, quantum spin models with high-order terms can have a phase diagram qualitatively different from their classical counterparts. In particular, at zero temperature they may have nonmagnetic ordered phases such as quadrupolar phase. These nonmagnetic phases have been a focus of attention in recent years. ${ }^{8}$

In order for a higher-order term to have a nontrivial contribution to the Hamiltonian, the spin must be larger than or equal to unity. Among the simplest $S=1$ cases, we consider the model with the highest symmetry since it probably provides us with a good starting point for developing a complete study of a wider range of models with lower symmetry. In the present article, therefore, we discuss the $S=1$ isotropic bilinear-biquadratic Heisenberg model

$$
H=-\sum_{(i j)}\left[J_{L} \boldsymbol{S}_{i} \cdot \boldsymbol{S}_{j}+J_{Q}\left(\boldsymbol{S}_{i} \cdot \boldsymbol{S}_{j}\right)^{2}\right]
$$

Since the biquadratic term in this Hamiltonian arises from the fourth-order perturbation of electron exchanges, it is usually smaller than the bilinear term that results from the second-order perturbation. However, it was pointed out ${ }^{10}$ that the bilinear term can be comparable with or smaller than the biquadratic one as a result of the cancellation of ferromagnetic and antiferromagnetic contributions, when we take various hopping terms into account.

For the one-dimensional case, a number of exact solutions and high-precision numerical calculations have established the character of most of the phases and the transition points. For the two- or higher-dimensional cases, on the other hand, our understanding largely depends upon mean-field-type approaches or semiclassical theories. ${ }^{11}$ A phase transition to a nonmagnetic ordered phase was predicted for a wide range of biquadratic models including the present model. The mean-field approximation ${ }^{12}$ was applied to the present model, resulting in there being a first-order phase transition from the paramagnetic phase to the quadrupolar phase (or the spin-nematic phase) when the biquadratic interaction is sufficiently large.

Since the mean-field-type approaches are usually accompanied by uncontrollable errors, a confirmation through rigorous proof or numerical calculations is necessary. In the classical model $(S=\infty)$ with small $J_{L} / J_{Q}$, it was rigorously proved $^{13}$ that the quadrupole moment is finite in some temperature range above the dipolar transition point. In the quantum case of $S=1$, the quadrupole moment was proved ${ }^{14}$ to be finite at zero temperature in some range of the parameter $J_{L} / J_{Q}$ in three dimensions. The range where this rigorous proof applies is not the same as, but smaller than, the quadrupolar region predicted by the mean-field arguments. In two dimensions, there is no rigorous proof of the existence of the quadrupolar phase.

We reported in previous work ${ }^{15}$ that the parameter space of positive $J_{Q}$ is divided by the two $\mathrm{SU}(3)$ points into three regions: ferromagnetic $(-\pi \leqslant \theta \leqslant-3 \pi / 4)$, antiferromagnetic $(-\pi / 2 \leqslant \theta \leqslant 0)$, and nonmagnetic $(-3 \pi / 4<\theta$ $<-\pi / 2$ ) regions. Here $\theta$ is defined by

$$
J_{L}=-J \cos \theta, \quad J_{Q}=-J \sin \theta \quad(J>0) .
$$

The nature of the nonmagnetic phase was not numerically identified in the previous work, although the mean-field theory predicted that it is the quadrupolar phase. In the present paper, we show for the model with negative $\theta$ that (1) the nonmagnetic phase is characterized by a finite quadrupole moment in two and three dimensions, (2) a phase transition to quadrupolar phase occurs at a finite temperature in three dimensions, and (3) the quadrupolar transition is of second order in contrast to the mean-field prediction. We also discuss the critical behavior of the three-dimensional system at finite temperature.

In the classical counterpart of the present model, the longrange order at zero temperature is always dipolar, i.e., ferro- 
magnetic or antiferromagnetic, except for the special case of $\theta=-\pi / 2$, where the dipolar degrees of freedom are noninteracting and disordered. In contrast, for the quantum model for $S=1$, it is argued based on a mean-field approximation ${ }^{12}$ that there is an intermediate phase between the antiferromagnetic region and the ferromagnetic region, and that this phase is characterized by a finite quadrupole moment. Because of the limitation of the mean-field-type theory, it always predicts a finite-temperature phase transition to the quadrupolar phase regardless of the dimensionality. This is of course wrong in one dimension. In two dimensions, too, the existence of a finite-temperature phase transition is very questionable because of the Mermin-Wagner theorem. Even at zero temperature, the existence of a finite quadrupole moment is not totally clear. Mathematically rigorous arguments, so far, have not established any long-range order in the intermediate parameter region.

In order to answer the question concerning the existence of quadrupole order, we performed Monte Carlo simulations using the loop algorithm proposed in the previous paper. ${ }^{15}$ The algorithm removes the ergodicity problem and considerably reduces the critical slowing down. The energy $E$, the dipole moment (i.e., magnetization) $M_{z}$, the staggered magnetization $N_{z}$, and the quadrupole moment were measured. We consider only the $z z$ component of the quadrupole moment in this article, which we denote by $Q_{z}$ :

$$
Q_{z} \equiv \sum_{i}\left(\left(S_{i}^{z}\right)^{2}-\frac{2}{3}\right) \text {. }
$$

The equal-time structure factors and the susceptibilities associated with these quantities were also measured. The system size ranges from $L=4$ up to $L=128$ for the twodimensional case and up to $L=64$ for the three-dimensional case. For each data point, we typically run the simulation for more than 10000 Monte Carlo steps.

For each system size in two dimensions, the thermal average of the absolute value of the quadrupole moment, $q$ $\equiv\left\langle\left|Q_{z}\right|\right\rangle / N$, converges to a certain finite value as the inverse temperature $K \equiv J / k_{\mathrm{B}} T$ increases. Here, the absolute value $\left|Q_{z}\right|$ is taken in the representation basis in which $Q_{z}$ is diagonalized. For any finite system, the convergence is exponential with some characteristic (imaginary) time scale. Although this characteristic time is larger for larger systems, the size dependence is weak. Therefore, we can extrapolate the data to the limit of $K=\infty$ without examining extremely low temperatures. After taking the zero-temperature limit numerically, we then take the infinite-system-size limit. The system size dependence is algebraic:

$$
q(L, K=\infty) \sim q(L=\infty, K=\infty)+a / L .
$$

This system size dependence is the same as that of the staggered magnetization in the antiferromagnetic Heisenberg model in two dimensions.

The quadrupole moment at zero temperature as a function of $\theta$ for various system sizes is plotted in Fig. 1, together with the extrapolation to infinite size. We now see that the quadrupole moment is finite in the intermediate phase as well as in the dipolar phases. In addition, it exhibits discontinuity

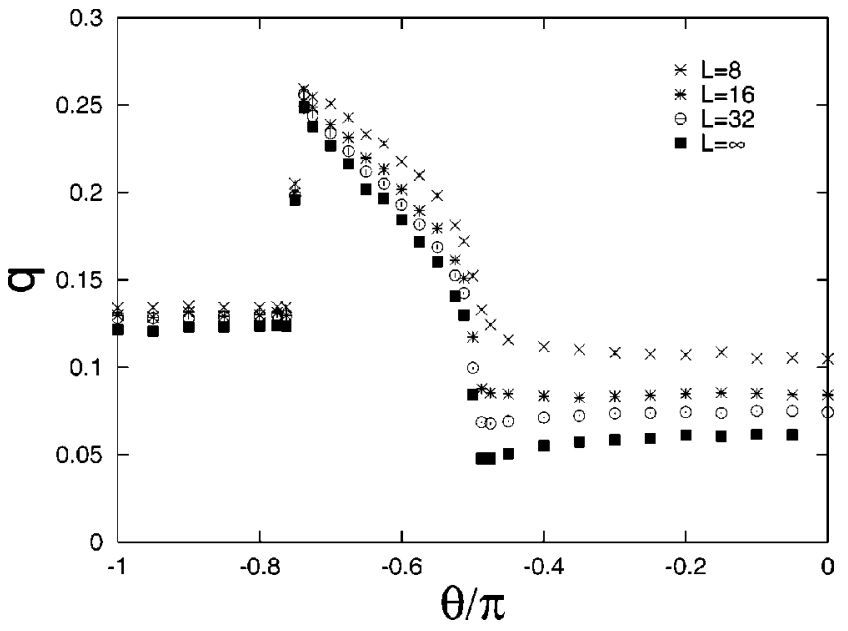

FIG. 1. The quadrupole moment at zero temperature in two dimensions.

at the two SU(3) points. Since the quadrupole moment is finite whenever the dipole moment is finite, it falls down to a finite value, not to zero, as we pass the phase boundary from the intermediate region to one of the two dipolar regions. Since the dipole moment is vanishing in the intermediate phase as we saw in the previous paper, ${ }^{15}$ the quadrupole moment is the characterizing order parameter for this phase.

In order to check the existence or absence of a phase transition at finite temperature, we have examined the specific heat. We have observed a broad peak at the temperature that roughly corresponds to the saturation temperature of the quadrupole moment. The peak height and width do not show a significant size dependence, indicating that it is not a phase transition but only a point where a relatively large number of free spins are frozen.

We plot in Fig. 2 the size dependence of the quadrupole moment as a function of system size, at various temperature in the case of $\theta=-0.6 \pi$. In this case, the peak in the specific heat is located at $K \sim 1.2$. In Fig. 2 , we see that the quadrupole moment shows the asymptotic size dependence

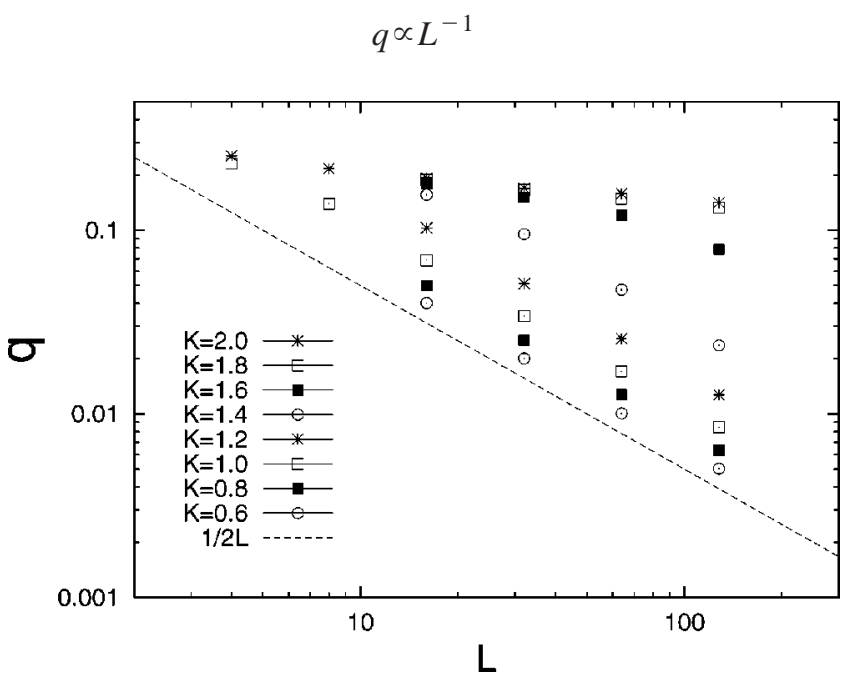

FIG. 2. The quadrupole moment as a function of the system size at $\theta=-0.6 \pi$ in two dimensions. 


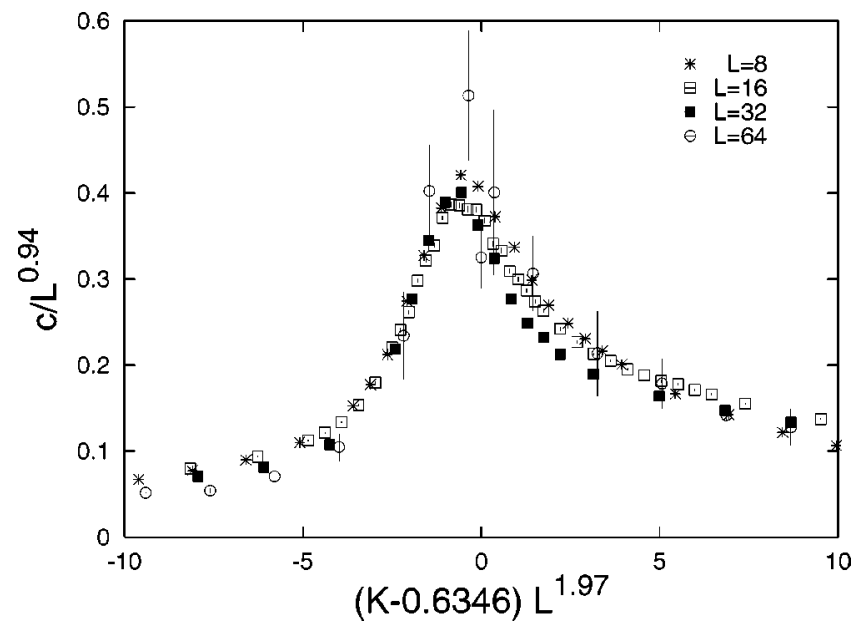

FIG. 3. Scaling plot of the specific heat at $\theta=-0.6 \pi$ in three dimensions.

down to the temperature $K \sim 1.6$. For a temperature lower than 1.6, the largest system size that we examined is not large enough to see the asymptotic behavior. The transition temperature of the Kosterlitz-Thouless-type (KT-type) phase transition is usually about $10 \%$ or $20 \%$ smaller than the peak temperature of the specific heat. Therefore, if there were a KT-type transition, we should be able to see a nontrivial algebraic decay for $K<1.6$, which we do not detect in Fig. 2. This indicates that there is no phase transition at any finite temperature.

For the system in three dimensions at zero temperature, we again observe three parameter regimes: ferromagnetic, quadrupolar, and antiferromagnetic, with exactly the same phase boundaries as those in two dimensions. Namely, the nature of the ground state changes at the two $\mathrm{SU}(3)$ points $\theta=-\pi / 2,-3 \pi / 4$. To see this in detail, we analyze the order parameters as in the two-dimensional case, the extrapolation to zero temperature, and then to the infinite system size. The behavior of the zero-temperature quadrupole moment as a function of $\theta$ is similar to the two-dimensional case, but the convergence to the infinite-size limit is faster. The quadrupole moment shows discontinuity at the two symmetric points. The zero-temperature phase diagram in three dimensions turns out to be exactly the same as that in two dimensions. We speculate that this is true for any dimension except for one dimension.

Having seen the long-range order at zero temperature in the intermediate quadrupolar regime, we now ask if there is a phase transition at finite temperature. Even in two dimensions, we have seen a broad peak in the specific heat and a crossover behavior from completely disordered states to partially ordered states as we decrease the temperature. This may be regarded as a precursor to the phase transition in higher dimensions. In fact, in the specific heat as a function of temperature, we see a much sharper peak in three dimensions than in two dimensions. The peak is not only sharp, but also shows clear size dependence, indicating a phase transition.

We can clearly see a strong correction to scaling, especially in Fig. 3. According to mean-field theory, ${ }^{12}$ this is a

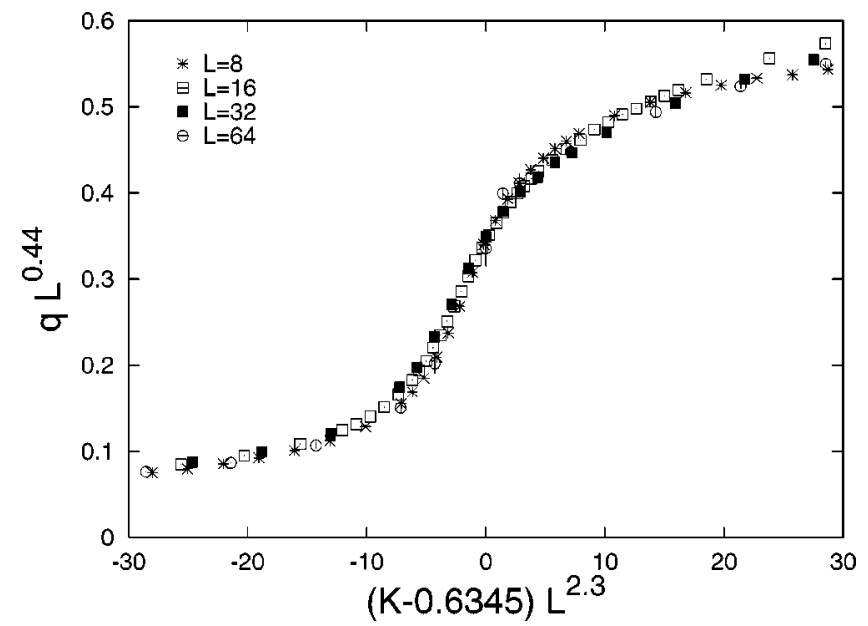

FIG. 4. Scaling plot of the quadrupole moment at $\theta=-0.6 \pi$ in three dimensions.

first-order phase transition. If this is the case, the peak height and width should be proportional to $L^{d}$ and $L^{-d}$, respectively. In other words, a finite-size scaling plot with exponents $d$ and $-d$ for the vertical scale and the horizontal scale, respectively, should work. Other quantities should also obey similar scaling forms with trivial exponents. We find that this is obviously not the case for any quantity. Instead, we assume the following finite-size scaling forms:

$$
\begin{gathered}
c=L^{2 y-d} \tilde{c}\left[\left(K-K_{c}\right) L^{y}\right], \\
q=L^{\beta y} \tilde{q}\left[\left(K-K_{c}\right) L^{y}\right],
\end{gathered}
$$

where $c$ is the specific heat. The best plots are obtained with

$$
y=1.97 \text { and } K_{c}=0.6346,
$$

for the specific heat, and

$$
y=2.30, \quad y \beta=0.44, \quad \text { and } K_{c}=0.6345 \text {, }
$$

for the quadrupole moment. The scaling plots are shown in Fig. 3 for the specific heat and in Fig. 4 for the quadrupole moment. The discrepancy among the estimates of indices may be due to a relatively large contribution of the nonsingular part to the specific heat. We have estimated the critical temperatures and indices at $\theta=-0.7 \pi$ in a similar fashion, and found that the critical indices are close to the corresponding ones for $\theta=-0.6 \pi$ quoted above. This fact suggests that they belong to the same universality class, as expected. Based on these results, we conclude

$$
y=2.15(20) \text { and } y \beta=0.46(4)
$$

for $-3 \pi / 4<\theta<-\pi / 2$.

The two $\operatorname{SU}(3)$ points $\theta=-\pi / 2$ and $-3 \pi / 4$ are of special interest, since the universality class of the critical point may be different from the one discussed above due to the higher symmetry. For these points of higher symmetry, we obtained better scaling plots than Figs. 3 and 4. The estimated critical temperatures are $K_{c}=0.6389$ (3) for $\theta=$ 


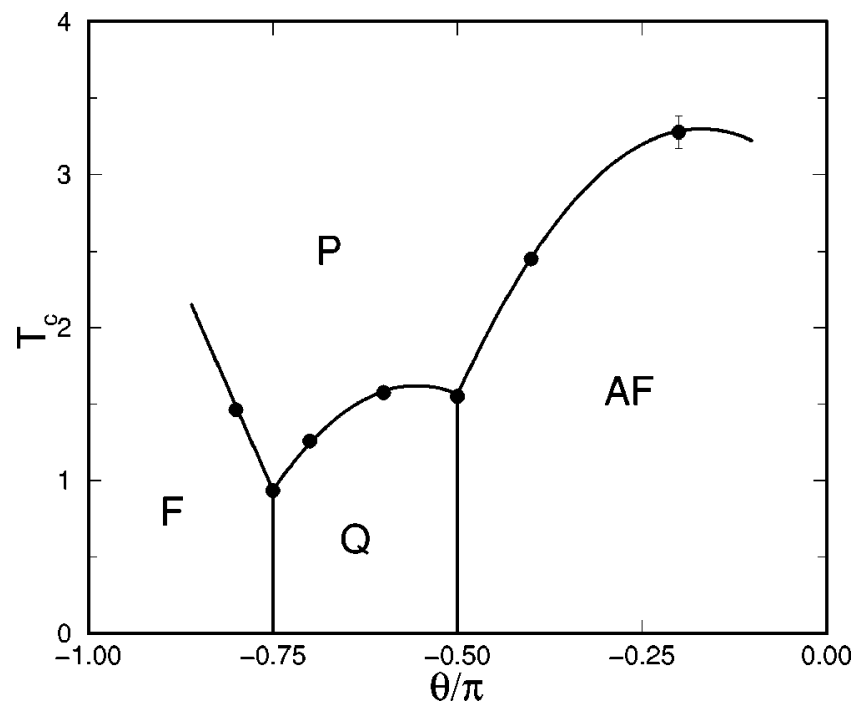

FIG. 5. The phase diagram of the three-dimensional model. Curves are mere guides to the eyes.

$-\pi / 2$ and $K_{c}=1.0724(4)$ for $\theta=-3 \pi / 4$. The critical indices for these two cases agree with each other, yielding

$$
y=1.82(5), \quad y \beta=0.48(1) .
$$

These results suggest that the critical points of the two SU(3) models belong to the same universality class and it is distinct from the one for the less symmetric cases although the difference in the indices is small. In Fig. 5, we summarize the estimated critical temperatures in the form of a $\theta-T$ phase diagram.

To summarize, we have studied the isotropic biquadratic
Heisenberg model in two and three dimensions for negative $\theta$. In two dimensions, we have identified the intermediate phase as the quadrupolar phase. The phase transition at a finite temperature has been excluded. In three dimensions, we have studied the finite-temperature properties as well as zero-temperature ones. At zero temperature, the phase diagram is exactly the same as that of the two-dimensional case. We have found that there is a finite-temperature phase transition not only in the ferro- and antiferromagnetic regimes, but also in the quadrupolar regime. In contrast to the meanfield prediction, the transition to the quadrupolar phase has turned out to be of second order. The critical indices are also estimated. While the two SU(3)-symmetric points belong to the same universality class, it is suggested to be distinct from the one for the less symmetric [i.e., SU(2)] models. Studies on the properties of low-lying excitations are still in progress and will be reported elsewhere. Less symmetric models with higher-order interactions may be more important than the present model from the practical point of view, since higherorder interactions in real magnets often arise from crystalline effects, which have lower symmetry. Studies on some of these models are also in progress.

The authors thank C. Batista, G. Ortiz, J. E. Gubernatis, and Y. Okabe for their useful comments. A part of N.K.'s work was done while he was staying at the University of Cergy Pontoise, France. He is grateful to H.-T. Diep for his hospitality. The computation was performed on SGI Origin 2800/384 at the Supercomputer Center, University of Tokyo, Institute of Solid State Physics, University of Tokyo. The present work is financially supported by a Grant-in-Aid for Scientific Research Programs (No. 11740232 and No. 12740232) from JSPS, Japan.
${ }^{1}$ M. Suzuki, Prog. Theor. Phys. 42, 1086 (1969).

${ }^{2}$ M. Blume and Y. Y. Hsieh, J. Appl. Phys. 40, 1249 (1969).

3 J. Kanamori, J. Appl. Phys. 31, 14S (1960).

${ }^{4}$ R. J. Elliott, A. P. Young, and S. R. P. Smith, J. Phys. C 4, L317 (1971).

${ }^{5}$ R. Settai et al., J. Phys. Soc. Jpn. 67, 636 (1998).

${ }^{6}$ P. Morin, J. Magn. Magn. Mater. 71, 151 (1988).

${ }^{7}$ F. J. Ohkawa, J. Phys. Soc. Jpn. 52, 1983 (1983).

${ }^{8}$ R. Shiina, H. Shiba, and P. Thalmeier, J. Phys. Soc. Jpn. 66, 1741 (1997).
${ }^{9}$ P. Morin, D. Schmitt, and E. T. de Lacheisserie, J. Magn. Magn. Mater. 30, 257 (1982).

${ }^{10}$ F. Mila and F.-C. Zhang, Eur. Phys. J. B 16, 7 (2000).

${ }^{11}$ N. Papanicolaou, Phys. Lett. A 116, 89 (1986); Nucl. Phys. B305[FS23], 367 (1988).

${ }^{12}$ H. H. Chen and P. M. Levy, Phys. Rev. B 7, 4267 (1973).

${ }^{13}$ A. Tanaka and T. Idogaki, J. Phys. Soc. Jpn. 67, 604 (1998).

${ }^{14} \mathrm{~K}$. Tanaka, A. Tanaka, and T. Idogaki (private communucation).

${ }^{15}$ K. Harada and N. Kawashima, J. Phys. Soc. Jpn. 70, 13 (2001). 